AGH DRILLING, OIL, GAS • Vol. $31 \cdot$ No. $2 \cdot 2014$

http://dx.doi.org/10.7494/drill.2014.31.2.253

\author{
Argentina Tătaru*, Florinel Şuţoiu*, Bolázs Sándor*
}

\title{
MATURE GAS FIELDS REHABILITATION CONCEPTS
}

\section{INTRODUCTION}

Some of the mature gas fields can not be exploited with attractive final recovery factors, through constrains of geological nature, technological or economical nature. In most of the cases, the geological elements are those who influence definitive the exploitation behaviour of the respective deposits, such that the existence of some traps due to the continuity and discontinuity character of the porous - permeable medium, or some reservoir parameters with low values, drastically reduce the extraction possibility of a larger volume out of the geological gas resource.

As well the exploitation performances of gas reservoirs, through various wells, are majorly affected due to deterioration and obstruction of the porous - permeable space from the well adjacent zone, during the drilling or opening the gas saturated layers. The existence of a skin factor with high value produces a increased flow resistance in the layer - well system, what is added to the resistance caused by hydrodinamic imperfection of the opening mode and degree of apperture of the production layer.

Liquid impurities quantity, accumulated in wells due to production with a flow rate smaller than the critical flow rate to entrane water through tubing, is another factor which produces an obstruction in the immediate nearness of wells. The surface infrastructure, properly undimensioned or with a affected integrity, generates a series of strangulations in the exploitation system, for example, reduced flow rates, inefficient separation, pressure loss. All these disruptive elements participates in different percentage in exploaitation behaviour of the respective reservoirs, with negative implications regarding well productivity.

* SNGN Romgaz SA Mediaş, Romania 
Exploitation performances of gas reservoirs are imposed by the main characteristics of the rock collector, but these parameters can be improved through stimulation operations, and their association with addequate extraction equipments and technologies, may lead to maximizing recovery of gases.

\section{GENERAL REHABILITATION CONCEPT}

Conceptually, the rehabilitation of mature gas reservoirs may be detected in three sequences: reservoir, well, surface facility. The intervention in one or another sequence, or concomitant intervention in all three sequences, is dictated by the technical reason economical reason tandem. In this sense, we elaborated a general rehabilitation scheme, presented in figure 1. and explained further on. Indifferent from approaching, the primary activity that begins on any rehabilitaion project, consist in evaluation of owned informations, which further generates, a set of new acquisitions opportune to redimensioning and improving the database.

Effectuation of 3D seismic and some complex geophysical investigations, of the latest generation, offers the support for a new vision about the reservoir model. Using the interpretation of 3D seismic and the different attributes obtained from it, in addition to petrophysical interpretation of geophysical loggs, allows the creation of a tridimensional model of the reservoir, from the structure point of view, the facies, total and net thickness, porosity, saturation and permeability. At the end of the 3D modelling process are estimated the geological resources, through volumetrical method. Geological resources can be evaluated also through material balance method, using the pressure/time function graphic, after previously the pressures were calculated using the mass conservation law, on the strenght of exploitation objectives production and gas properties.

Verifying the previous estimations through numerical simulation, by construction of some dynamical models for every hydrodynamical unit, offers the perspective of an ample approaching of evaluation of resources and gas reserves. Using this model is extremely indicated, because it considers all the available informations about the reservoir, as much as static behaviour and dynamical. The finality of numerical simulation consist actually from confirmation of initial resource for each expoitation objective, defining reserves and elaboration of a developing plan of the reservoir.

For a complete image on the entire gas structure, it is necessary also the acces of exploitation parameters, evaluation of well productivity and elaboration of some sensitivity analisys in the points of interest or problems, from the trail well - surface facility, in this way beeing detected the zones which are the best suitable for rehabilitation.

The newly identified reserves, from undraines or less drained zones, will be verified and revaluated through a working program, which objectives are the optimization and maximizing of gas production, respectively increasing the recovery factor. The elaborated strategies will be subjected to economical analysis, and if this finds that they could develop under rentability conditions, the respective approaches will become viable. 


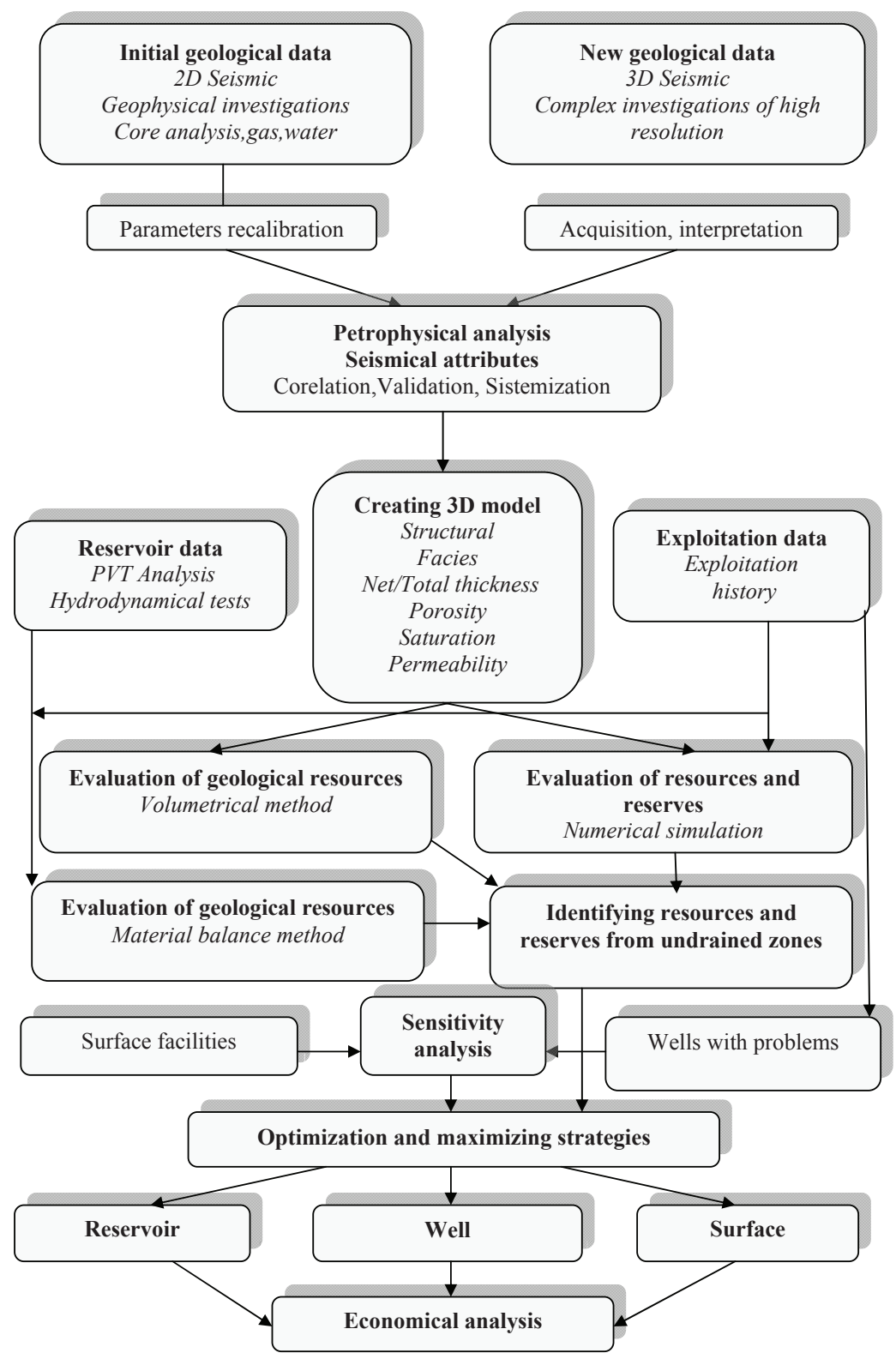

Fig. 1. General rehabilitation scheme of mature gas reservoirs

The scheme presented in figure 1 contain the main stages which could be covered within general rehabilitation process of mature gas reservoir, starting from the existence of a database which contains geological informations, reservoir and exploitation. 


\section{GEOLOGICAL MODEL RECONSIDERATION}

For the selection of the most suitable exploitation mode of the gas reservoirs, it imposes, that after defining the reservoir formes from seismical interpretation, to evaluate the geological model.

In this sense it is necessary:

- determination of the reservoir main facies, specifying the characteristics of porosity, permeability, seal role;

- specification of distribution in space and lithological corelation, upon geophysical loggs;

- dividing the reservoir ensemble into many units;

- quantification of tridimensional evolution of the reservoir characteristics for each unit: total and net thickness, lithology, clay content, porosity, permeability;

- evaluation of the expansion and dynamic role for some lithological packs: drainages, sealings.

Achieving this step assumes many stages:

- well core analysis and loggs reference, as well the definition of a sedimentary model;

- uncored well loggs analysis and the expansion of the sedimentary model through vertical sections, specifying the corelations between the wells;

- stratigraphical and lithological interpretation on seismical data, through extrapolation, if data are lent to the reservoir ensemble model;

- integration of dynamic data (interference tests, production tests, pressure measurements) and validation of geological model.

In case of mature gas fields, reconsideration of the geological model, reevaluation of gas resources and reserves in different exploitation moments, represents a real support for further rehabilitation approach.

Maximizing the reservoir view using advanced seismical interpretations, based on 3D and 4D seismical monitoring, but also on complex technologies for viewing new volumes of resources, permits a reevaluation, sometimes spectacular of the geological model. The new investigation technologies for wells, capable to highlight the thinest natural layers saturated with gas, offers a new chance for mature reservoirs as well, representing the foundation for a reevaluation of gas resources and reserves.

The tridimensional modeling allows the volumetrical calculation of gas resources, using 3D seismical interpretation and the different attributes extracted within, but also on the obtained informations from petrophysical interpretations of well geophysical loggs, but also from core analysis. The main characteristics of the reservoir rock are reinterpreted for improving the reservoir geological model.

The informations regarding the reservoir type, fluid distribution and reservoir rock characteristics, allows the geological modeling and subsequent evaluation of the gas resources. 
Recently appeared a series of softs which allows volumetrical calculation of gas resources through tridimensional modeling and whom essentially go through the same stages for building the 3D model:

- import available data

- performing petrophysical interpretation and the digital data set are imported to the program; analyzing the complete set of geophysical logging performed in open or cased hole and core;

- performing seismical cube interpretation data and identifying the marks

- corelations

- performing the corelation of all the existing marks, identified from electrical loggs and seismical investigation;

- structural - stratigraphical frame

- creating 3D models for each exploitation objective, based on horizonts from seismical interpretations and marks interpreted through stratigraphic corelation of electrical loggs;

- facies modeling

- facies modeling with defining the detailed sedimentological model

- properties modeling

- modeling the reservoir properties, total and net thickness, porosity, saturation, clay volume, permeability, for populate the 3D model;

- volumetrical calculation

- calculating the initial geological resource for each zone, with the created 3D model.

Tridimensional modeling presumes in fact the creation of 6 models, based on all the available geological data, arised from older or more recent acquisitions, correlated and systematized.

- Structural model - based on seismical interpretation and stratigraphical correlation of electrical loggs are created structural grids, lately are generated structural maps and isopach maps for each exploitation objective;

- Facies model - based on clay volume analysis obtained from petrophysical interpretations of the geophysical loggs, as well based on seismical attributes are created lithological models for each expoitation objective; from the clay volume analysis can be determined the average clay and sand content distribution, but also a series of lithological histograms and variograms over the three directions: $\mathrm{x}, \mathrm{y}, \mathrm{z}$;

- Total/net thickness model - based on the facies model are created thickness models, lately are generated net thickness maps for each exploitation objective; the lithology classes are reduced to two groups: reservoir and non-reservoir, assigning to each group a total/net thickness value;

- Porosity model - based on the clay volume model are created porosity models, lately are generated distribution maps of porous volume for each exploitation objective;

- Fluid saturation model - based on petrophysical interpretations data of geophysical loggs, through correlation with porosity models are created fluid saturation models;

- Permeability model - based on porosity models are created permeability models, in condition of existing of a direct relation between the two parameters. 


\section{RESERVOIR REHABILITATION}

From the point of view of the reservoirs effective rehabilitation can be accesed new drillings, existent wells deepening, reperforations and additions of undepleted new layers and not least stimulation operations of the reservoir.

Drilling development in mature gas fields presume a number of challenges, but the technological modifications in this area offer new benefits which include drilling cost reduction and increased investment recovery.

Underbalanced drilling represents one of the best technics when drilling in low pressure reservoirs, mature reservoirs or naturally fractured, the major benefits associated to this technology being bound to the maximizing of well productivity or reduction drilling problems.

If underbalanced drilling follows the minimization of productive layer deterioration and to increase advancement velocity, pressure controlled drilling has the purpose to decrease the unproductive times, respectively cost decreasing, through drilling problems reduction (circulation loss, erruptive manifestations, string stuckings).

Stimulation operations of gas reservoirs offers the possibilty of improvement of some porous - permeable parameters around the well, respectively increasing the collective rock permeability and implicit increasing the flow capacity. Clearing the existent flow channels or creating some new channels, generates the development of productive potential of stimulated reservoirs.

The most used stimulation methods are the acidization and hydraulic fracturing. Perceived as a sure method for cancelling or reducing the flow resistances of gas from layers into the well, hydraulic fracturing could be succesfully applied to mature gas fields. Creating new fractures or extending the natural ones permits a increasing of gas influx into porous - permeable space from the well adjacent zone, which conducts on longer term, to a superior recovery of gas resources.

The purpose of a stimulation operation through hydraulic fracturing is to create a zone with high connectivity compared to reservoir permeability from a certain distance from well, which will determine a increasing of productivity of the respective well. Maintaining the fracture, and also the increased connectivity zone, is realised through filling with a special material.

Hydraulic fracturing is a physical process in which the layer surrenders on the minimal resistance planes, under the effect of pressure of the fluids pumped into the well. The reason of hydraulic fracturing is to realise a increased gas influx into the layer zone around the well, on a certain radius equivalent with the created fracture radius.

The correct attitude regarding this type of stimulation is to intervene with an optimal treatment which maximizes the profit. The optimal treatment, however, is different from one reservoir to another, from one layer of the reservoir to another, and therefore the answer given by production of each fractured zone will be different.

Selecting the best candidates for hydraulic fracturing must have in consideration the tectonical, lithological, geological, reservoir and technological conditions, which in equal measure, will mark the final results. For example, a agitated tectonical history of the zone in which the reservoir was formed, the existence of impermeable barriers in the top and bottom of hydrodynamic unit, unadequate values of reservoir parameters, lack of adherence of the cement between casing and formation, will indisputable affect the succes of a this type of stimulation operation. 
Certainly, the succes or insucces of the hydraulic fracturing operations will be decided also from the availability and judicious use of informations necessary for optimizing the planning this type of stimulation. It is needed a considerable number of data for planning a hydraulic fracturing operation, but their correctness will constitute an important aspect in obtaining the most efficient simulation model.

\section{SURFACE FACILITIES REHABILITATION}

Modernizing the surface facilities (technological installations, drying and compressing capacities, monitoring and real time transmission of production parameters), offers a interesting perspective about optimization and maximization of gas production.

Indifferent from the exploitation regime of gas reservoirs, the possibility of resource recovery is influenced by geological, technical and economical factors, and in this context, for setting the final recovery factors, it is necessary an analysis of the customizable conditions of each resevoir.

The detailed model on which are determined the productive performances of a gas reservoir has in view the following system:

draining zones $\rightarrow$ wells $\rightarrow$ chockes $\rightarrow$ adduction pipeline $\rightarrow$ collecting pipeline $\rightarrow$ $\rightarrow$ compression station $\rightarrow$ main pipeline

Modifying any parameter which characterize the components of this system, is influencing the exploitation behaviour of the reservoir.

As the exploitation process is ongoing, appears as an effect the natural decline of reservoir static pressure and the production performances. If at the beginning of the exploitation, the flow rates decline is exclusively influenced by reservoir static pressure, towards the end of the exploitation, it is influenced by the reservoir pressure decline such as by the collecting system pressure.

If in case of depleted reservoirs if there is no intervention for reducing the collecting system pressures for wells, de flow decline will have a parallel evolution with the reservoir pressure decline.

The reservoir reaction, in sense of increasing the flow over decreasing the collecting pressure, is essential for a decision effect, regarding the compression type and its placement location. A viabil solution is to set a compressor with low suction pressure, preferably, as close to the well-head as possible, for removing the inconvenient of supplementary pressure lost, in the collecting system, upstream the compressor.

Using this type of compressor is reccomended from the following considerations:

- large limits of discharge/suction pressure ratio

- reduced maintenance and exploitation costs

- disposition in a unique skid, with extremely easy possibility of ulterior reassembling in another location

- there is no intermediate cooling of the gas

- reduced exploitation personnel needed. 
The economical reasons for implementing a compression project are determined by the acquisition and installation and operating costs, production profile resulting from dropping the collecting system pressure. There will appear two questions, whose answers may constitute the decisional base for implementing one alike project. How many wells will respond at the pressure drop? Which is the lowest pressure, so that the flow and additional recovery justifies the investment effort?

In the first case, the well's answers to pressure drop must be simulated, which presumes using some diverse methodologies, through a multitude of soft's available, in present moment. Generally, the simulation shows that the well's respond with different ranks to pressure drop, according to reservoir characteristics. Wells with similar reservoir characteristics will offer after the simulation, appropiate final recovery factors, which facilitates grouping the wells regarding the selection of the best modelling version, and implementing the compression.

Generally, a simulation based on nodal analysis involve some limitations. Because nodal analysis assumes stationary flow, it is difficult to stimulate the accumulation model of the liquids in wells, which presumes a tranzitory behaviour, and in this case it regards velocity and critical flow rates.

Another type of simulation uses the material balance for modelling the reservoir and a soft for modelling the production grid. The combination between the two approaches is considered an intergrated model of production and uses boundings and data exchanges in the two simulation systems.

The pressure drop by implementing field compression and at the well-head, applied to those reservoirs with permisive coefficients to flow resistivity, induce a superior gas resource recovery, and in this context could be considered one of the pertinent rehabilitation versions of mature gas reservoir.

\section{CONCLUSIONS}

The rehabilitation of mature gas fields represents in fact a complex of technical, economical and management strategies, which purpose is maximizing the production and implicit increasing the recovery factor of gas resources, in rentability conditions.

During the exploitation, after recording a considerable production history and implicit a considerable depletation, it is necessary to evaluate the maintenance or the exchange of the exploitation strategy. The approaches in this sense must be from the prespective of improving the value of mature gas fields.

Reasoning the rehabilitation opportunity of mature gas fields, is given by the existence of some still attractive volumes, which could not be extracted due to different constrains, presuming the identification of the most valuable solutions regarding the realization of some levels of exploitation as high as possible.

Although the rehabilitation opportunity of mature gas fields is obvious and unanimously accepted, the effective implementation of the concept represents a complex intervention. This 
fact is due to the diversity of gas resources, which presumes the elaboration of some particular rehabilitation models, adapted in part to each reservoir capability.

\section{REFERENCES}

[1] Tătaru, A.: Contributions to mature gas fields rehabilitation, PhD. Thesis, Petrol-Gas University, Ploieşti, 2012

[2] Ştefănescu, D.P.: Introduction into mature gas fields rehabilitation, Theory and case studies, Lucian Blaga University Publishing, Sibiu, 2011. 